\title{
Comportamentos sedentários e sua relação com os componentes da síndrome
}

\section{metabólica em quilombolas}

\author{
Sedentary behaviors and their relationship with the metabolic syndrome components in \\ quilombolas \\ Comportamientos sedentarios y su relación con los componentes del síndrome metabólico en \\ quilombolas
}

Claudio Bispo de Almeida

ORCID: https://orcid.org/0000-0001-9486-7163 Universidade do Estado da Bahia, Brasil E-mail: cbalmeida@uneb.br

Lorena Andrade Nunes

ORCID: https://orcid.org/0000-0002-7453-7666 Universidade Estadual do Sudoeste da Bahia, Brasil E-mail: lorenunes2@gmail.com

Ricardo Franklin de Freitas Mussi

ORCID: https://orcid.org/0000-0003-1515-9121 Universidade do Estado da Bahia, Brasil E-mail: rimussi@yahoo.com.br Cezar Augusto Casotti

ORCID: https://orcid.org/0000-0001-6636-8009 Universidade Estadual do Sudoeste da Bahia, Brasil E-mail: cacasotti@uesb.edu.br

\begin{abstract}
Resumo
Os comportamentos sedentários (CS) podem estar associados aos componentes da síndrome metabólica (SM) em quilombolas. O objetivo deste estudo é investigar a associação entre os CS, tempo sentado e tempo de TV, com os componentes da SM. Trata-se de um estudo epidemiológico, transversal, de base populacional, com quilombolas residentes na microrregião de Guanambi, Brasil. Os dados foram obtidos por meio de entrevistas, mensurações antropométricas, aferições de pressão arterial e coleta sanguínea. Realizaram-se as análises: descritiva; ANOVA oneway; MANOVA; Análise de Componente Principal (ACP); e comparação pelo BoxPlot. Os resultados indicam que, entre os 850 quilombolas, prevaleceram: do sexo feminino $(61,2 \%)$; com menor renda (79\%); e com tempo de TV $\geq 5 \mathrm{~h}(69,7 \%)$. Na ACP, os componentes da SM não apresentaram associação com os CS. Entretanto, a ANOVA mostrou que o tempo de TV, entre os idosos, se associou $(\mathrm{p}<0,05)$ com circunferência de cintura $(\mathrm{CC})$, apresentando diferença entre os tempos de quatro horas e $\geq 5$ horas. Conclui-se que não houve associação entre CS e componentes da SM. Entretanto, em idosos quilombolas, a CC pode ser um indicativo de saúde aplicável no monitoramento e prevenção da SM.
\end{abstract}

Palavras-chave: Síndrome metabólica; Tempo de tela; Grupo com ancestrais do continente africano; Grupos étnicos.

\begin{abstract}
Sedentary behaviors (SB) may be associated with components of the metabolic syndrome (MS) in quilombolas. The aim of this study is to investigate the association between SB, sitting time and TV time, with the components of MS. This is an epidemiological, cross-sectional, population-based study with quilombolas living in the microregion of Guanambi, Brazil. Data were obtained through interviews, anthropometric measurements, blood pressure measurements and blood collection. Analyzes were performed: descriptive; One-way ANOVA; MANOVA; Principal Component Analysis (PCA); and comparison by BoxPlot. The results indicate that, among the 850 quilombolas, the following prevailed: female (61.2\%); with lower income (79\%); and with TV time $\geq 5 \mathrm{~h}(69.7 \%)$. In PCA, the components of MS were not associated with SB. However, the ANOVA showed that the time spent on TV, among the elderly, was associated $(p<0.05)$ with waist circumference $(\mathrm{WC})$, with a difference between the times of four hours and $\geq 5$ hours. It is concluded that there was no association between SB and MS components. However, in elderly quilombolas, WC can be an indication of health applicable in the monitoring and prevention of MS.
\end{abstract}

Keywords: Metabolic syndrome; Screen time; African continental ancestry group; Ethnic groups. 


\section{Resumen}

Los comportamientos sedentarios (CS) pueden estar asociados con componentes del síndrome metabólico (SM) en quilombolas. El objetivo de este estudio es investigar la asociación entre CS, tiempo sentado y tiempo frente a la televisión, con los componentes de la SM. Se trata de un estudio epidemiológico, transversal y poblacional con quilombolas que viven en la microrregión de Guanambi, Brasil. Los datos se obtuvieron a través de entrevistas, medidas antropométricas, medidas de presión arterial y recolección de sangre. Se realizaron análisis: descriptivos; ANOVA unidireccional; MANOVA; Análisis de componentes principales (ACP); y comparación por BoxPlot. Los resultados indican que, entre los 850 quilombolas, predominó: femenino (61,2\%); con menores ingresos (79\%); y con tiempo de TV $\geq 5 \mathrm{~h}(69,7 \%)$. En ACP, los componentes de MS no se asociaron con CS. Sin embargo, el ANOVA mostró que el tiempo dedicado a la televisión, entre los ancianos, se asoció ( $\mathrm{p}<0.05$ ) con la circunferencia de la cintura (CC), con una diferencia entre los tiempos de cuatro horas $\mathrm{y} \geq 5$ horas. Se concluye que no hubo asociación entre los componentes CS y MS. Sin embargo, en los quilombolas ancianos, la CC puede ser un indicador de salud aplicable en el seguimiento y prevención de la MS.

Palabras clave: Síndrome metabólico; Tiempo de pantalla; Grupo de ascendencia continental africana; Grupos étnicos.

\section{Introdução}

Os comportamentos sedentários (CS) são caracterizados pela realização de atividades nas posições sentada ou deitada, com gasto energético próximo aos níveis de repouso (Meneguci et al. 2015; Gibbs, Hergenroeder, Katzmarzyk, Lee, \& Jakicic, 2015; Bull et al. 2020). Neste sentido, atividades corriqueiras, como assistir televisão, usar computador, uso de veículos automotores, dentre outras, configuram CS. Tais comportamentos, mantidos por períodos prolongados, independente dos níveis de atividade física, têm sido associados a enfermidades e condições desfavoráveis de saúde da população, a exemplo das doenças e riscos cardiometabólicos (Gibbs et al. 2015; Figueiró et al. 2019), obesidade (Meneguci et al. 2015) e aumento do risco de mortalidade por todas as causas (Rezende, Rey-López, Matsudo, \& Carmo, 2014; Bull et al. 2020).

Destarte é importante diferenciar CS de atividade física, pois são constructos diferentes e que possuem efeitos distintos (Meneguci et al. 2015; Bull et al. 2020). Logo, ser sedentário, ou possuir baixos níveis de atividade física não são sinônimos de CS, neste sentido que surge a recomendação de incentivar a prática de alguma atividade física e de se reduzir os CS (Bull et al. 2020).

Estudos realizados com populações negras encontraram diferentes prevalências de CS, com variação de $14,79 \%$ a 56,89\% (Micklesfield et al. 2017; Matthews et al. 2014) e médias elevadas de tempo total gasto em CS (Atiase et al. 2015), além de associações dos CS com obesidade (Matthews et al. 2014; Atiase et al. 2015), risco metabólico (Atiase et al. 2015), hipertensão arterial (Cordovil \& Almeida, 2018), níveis de glicose (Atiase et al. 2015), hipertrigliceridemia (Dickie, Micklesfield, Chantler, Lambert, \& Goedecke, 2016), acidente vascular cerebral isquêmico (Owolabi et al. 2017) e risco de mortalidade por todas as causas (Matthews et al. 2014).

Dentre os perfis populacionais negros brasileiros, emergem os agrupamentos remanescentes quilombolas. Estas comunidades se caracterizam pela presença de pessoas de ancestralidade negra, atributos sociais, culturais e religiosos específicos, predominantemente residentes em localidades rurais distantes, sem a obrigatoriedade de ascendência em grupos de fugidos do sistema escravagista, mas, marcadamente insurgentes a opressão racista (Texeira Mussi, Mussi \& Araújo, 2020). Também são definidas pela auto atribuição, diante de relações territoriais específicas, trajetória sociohistórica própria, laços de parentesco comunitário e prática de cultura agrícola de subsistência (Mussi \& Petroski, 2019).

Tanto a legitimidade quanto a legalidade quilombola representam importantes marcos afirmativos na comunidade negra (Mussi et al. 2020a). No entanto, sabe-se que esta população ainda sofre impacto negativo dos resquícios oriundos do preconceito e descaso social, os quais podem ser evidenciados pelo regular estado de vulnerabilidade social em que vivem (Araújo et al. 2019; Pauli, Bairros, Nunes, 2019; Melo \& Garcia, 2019; Almeida, Santos, Vilela \& Casotti, 2019; Pereira, Mussi \& Rocha, 2020).

Neste contexto, é importante reconhecer que a associação entre vulnerabilidade e as relações sociohistóricas de 
dominação, intimidação e manipulação resultam em indicadores de saúde preocupantes (Mussi et al. 2020a).

O Nordeste brasileiro e o Estado da Bahia, são regiões que ainda mantém dificuldades no acesso as políticas públicas e condições socioeconômicas restritivas, possuem o maior quantitativo de quilombos certificados (Matos, Vasconcelos, Rocha \& Mussi, 2021). Além disso, as comunidades quilombolas estão localizadas predominantemente em áreas rurais (Almeida \& Casotti, 2021; Pereira et al. 2020; Araújo et al. 2017), regiões que ainda são marcadas pela frágil efetividade das políticas públicas, com possível reflexos negativos no estilo de vida e condições de saúde.

Os CS podem ser considerados como parte do estilo de vida de populações afrodescentes (Almeida \& Casotti, 2020). E, em populações quilombolas, a característica rural, marcada por atividades laborais ativas, tem ampliado o acesso a tecnologias, a exemplo do uso de celulares, veículos automotores e, como destaca Sousa et al. (2019), ampliou-se a utilização de aparelhos de TV por conta da expansão da energia elétrica nestas comunidades. Desta forma, por exemplo, o tempo de TV, que é um tipo de CS, tende a ser aumentado, e com ele a possibilidade de danos à saúde, independente do nível de prática de atividade física.

Assim, observa-se que, as comunidades remanescentes de quilombos, também estão expostas a CS excessivos (Cordovil \& Almeida, 2018; Soares \& Barreto, 2015; Mussi, Queiroz \& Petróski, 2018), o que permite compreender o quadro epidemiológico deste grupo populacional. Ressalta-se que os CS sofrem influências de aspectos socioculturais, como por exemplo, as condições de vida, trabalho, acessibilidade, ao modo de vida singular que essas comunidades rurais, possuem desigualdades que são estabelecidas, sobretudo, pelo racismo institucional existente na sociedade brasileira (Dimenstein et al. 2020).

Destarte, apesar de não serem encontradas análises sobre a relação entre CS e Síndrome Metabólica (SM) em quilombolas (Almeida \& Casotti, 2021), alerta-se para os impactos negativos da alta prevalência da SM para a saúde da população. Estudos anteriores com esta mesma população encontraram prevalência de SM igual 25,8\% (Mussi et al. 2020b; Mussi \& Petroski, 2019). Entretanto, maior prevalência foi encontrada em quilombolas adultos e idosos piauienses, com prevalência de SM de 55,4\% (Aragão, Bós \& Souza, 2014).

Em estudo sobre simultaneidades dos componentes da SM em quilombolas baianos (Mussi et al. 2020b) encontrou-se alta presença de simultaneidades aumentadas, e a hipertrigliceridemia foi o componente mais recorrente, e a maior simultaneidade foi associada ao trabalho remunerado e a menos de cinco anos de escolarização. Outro estudo, transversal, associou a SM em quilombolas ao sexo feminino, faixa etária maior ou igual a 40 anos, má qualidade do sono, excesso de peso corporal e obesidade (Mussi \& Petroski, 2019).

Diante do exposto, este estudo teve como objetivo investigar a associação entre os CS, tempo sentado e tempo de TV, com os componentes da SM.

\section{Metodologia}

Trata-se de um estudo epidemiológico, do tipo transversal (Almeida Filho \& Barreto, 2017), desenvolvido com residentes em comunidades remanescentes de quilombos da microrregião de Guanambi, Estado da Bahia, Brasil, no período de abril a novembro de 2016. Esta microrregião está situada na região Sudoeste do Estado e é constituída por 18 municípios e contava com 42 quilombos certificados durante o período das coletas, distribuídos em 10 destes municípios (Brasil, 2016). Tal população se caracteriza geograficamente como população rural, caracterizada como quilombo contemporâneo.

Frente à indisponibilidade de informações oficiais relacionadas a esta população, a estimativa populacional foi realizada ao se considerar 80 famílias por quilombo (Secretaria de Políticas de Promoção da Igualdade Racial da Presidência da República [SEPPIR], 2010), com dois adultos (>18 anos) por família, o que totalizou 6.720 quilombolas nesta faixa etária.

O cálculo amostral adotou correção para população finita, prevalência de $50 \%$ para desfecho desconhecido, intervalo 
de confiança de 95\%, erro amostral de cinco pontos percentuais, efeito do desenho 1,5 vez para conglomerado em um estágio, acréscimo de $30 \%$ para recusas e $20 \%$ para perdas e confundimento, determinou-se amostra mínima de 818 quilombolas. Este número foi suficiente para atingir a quantidade mínima da amostra, de forma a permitir a extrapolação dos resultados para a população.

Para se atingir a amostra mínima necessária, primeiro foi realizado um sorteio aleatório simples dos conglomerados (comunidades), constantes na lista das 42 comunidades certificadas. 17 foram sorteadas, entretanto somente 14 aceitaram participar do estudo, o que foi suficiente para atender os critérios amostrais estabelecidos. Em seguida, foi feita a identificação de todos os residentes nas comunidades sorteadas, por meio de lista fornecida pelas respectivas associações comunitárias.

Todos os quilombolas ( $\geq 18$ anos de idade) que residiam nos quilombos sorteados foram considerados elegíveis para o estudo. Foram critérios de exclusão, na pesquisa em geral: nas entrevistas, aqueles que apresentaram deficiência cognitiva ou de comunicação independente; e nas mensurações, os que se encontravam acamados, com amputação ou com engessamento de qualquer segmento corporal, além de mulheres gestantes ou nutrizes.

As perdas amostrais caracterizaram-se pela ausência de qualquer informação relativa a alguma medida, dados de exame ou ausência de resposta em questão da entrevista e as recusas pela não participação, que mesmo diante dos esclarecimentos relativos à pesquisa e convite para apresentar-se nos dias em que as atividades de coleta foram desenvolvidas em sua comunidade, optaram por não comparecer em nenhum dos encontros.

Assim, as 14 associações de moradores informaram a presença de 1.025 adultos e idosos residentes nas comunidades remanescentes de quilombo durante o período das coletas. Todos foram convidados a participar do estudo e informados sobre os seus aspectos, garantindo-se igual probabilidade de participação. Destes, 850 compareceram voluntariamente às atividades e aceitaram participar da pesquisa.

Para o presente estudo, foram excluídos das análises aqueles que: não continham dados dos CS; apresentaram menos de duas horas de CS; e menos de três componentes da SM. Desta forma, o tempo sentado apresentou uma amostra geral igual a 791, entretanto, como as análises não deram significância estatística, detalhamos somente o tempo de TV. Assim, entre os 850 quilombolas, o n geral do tempo de TV foi 746, e depois de retirados aqueles que não apresentam este CS maior ou igual a $2 \mathrm{~h}$, o n passou para 622. Na estratificação por grupo etário obteve-se o tempo de TV em: 291 adultos e em 64 idosos (Tabela 4).

Os dados foram obtidos em sistema de mutirão, em dias previamente agendados pelas associações de moradores das comunidades remanescentes de quilombos em comum acordo com os residentes. Em cada uma das comunidades, as coletas ocorreram em até três visitas. Para isso, foi composta uma equipe, previamente treinada, com 15 membros, considerando-se a seguinte configuração: um antropometrista treinado e experiente, uma pessoa com habilitação para coleta sanguínea por punção venosa e 13 entrevistadores padronizados para aplicar o questionário estruturado e mensurar a pressão arterial. Durante as entrevistas, realizadas face a face, os pesquisadores faziam as perguntas e anotavam as respostas dos participantes em um formulário.

A pressão arterial, sistólica (PAS) e diastólica (PAD), foi aferida em duplicidade. A primeira aferição foi realizada cinco minutos após o início da aplicação da entrevista, para garantir repouso inicial mínimo de cinco minutos, e a segunda ao final desta. Utilizou-se esfigmomanômetro semiautomático, da marca Omron, modelo HEM-742INT, com o manguito para adultos, e ajustado ao perímetro do braço do indivíduo, 2-3 centímetros acima da fossa cubital.

Em seguida, as medidas antropométricas foram coletadas segundo recomendações da International Society for the Advancement of Kinanthropometry - ISAK (Stewart, Marfell-Jones M, Olds \& Ridder, 2011). Para isso, utilizou-se balança digital previamente calibrada, da marca Omron, modelo hbf-514c, com resolução de 100 gramas e capacidade de 150 kg, para aferir a massa corporal. A estatura foi obtida por meio de estadiômetro portátil de alumínio, da marca Sanny, modelo caprice, com resolução de $0,1 \mathrm{~mm}$. A circunferência de cintura $(\mathrm{CC})$ foi obtida por meio de uma fita antropométrica metálica, da marca 
Sanny, modelo sn-4010, com dois metros de comprimento e resolução de $0,1 \mathrm{~mm}$, horizontalmente ao solo.

A coleta, armazenamento, transporte e processamento das amostras de sangue seguiram as normas da Sociedade Brasileira de Patologia Clínica e Medicina Laboratorial (2014). As amostras foram coletadas com os indivíduos em jejum noturno (mínimo de 08 horas) por meio de punção venosa de vasos da região antecubital. Foram tomados os cuidados com a antissepsia local da pele e foram evitados os vasos lesionados, avermelhados e próximos de áreas previamente infectadas. Utilizaram-se agulhas descartáveis, que foram descartadas em local específico após o uso.

Tubos a vácuo foram utilizados para armazenamento e posterior análise das amostras sanguíneas de glicemia (5 mL, seco, com fluoreto de sódio e EDTA), triglicérides e da fração HDL do colesterol (4mL, com ativador de coágulo). As amostras foram rotuladas com o número referente a cada participante e estocadas em recipiente com gelo para transporte e posterior análise laboratorial.

O processamento do sangue foi realizado em laboratório de análises bioquímicas. Para separar o soro do plasma, o sangue foi centrifugado a $3.000 \mathrm{rpm}$ por 10 minutos (Centrífuga 80-2b, Centribio, São Paulo) e o sobrenadante coletado e transferido para tubos de plástico e armazenado a -20oC para posterior análise. As medidas bioquímicas (glicose, triglicérides e colesterol) foram realizadas pelo método enzimático colorimétrico automatizado (Cobas Mira Plus, Roche®).

As variáveis categóricas utilizadas neste estudo foram: sexo (masculino; feminino); faixa etária (18 a 59 anos; $\geq 60$ anos); renda familiar mensal ( $\leq$ um salário mínimo; > um salário mínimo), sendo considerado o valor do salário mínimo igual a $\mathrm{R} \$ 880,00$ em 2016, ano de realização da coleta de dados. Os comportamentos sedentários avaliados foram o tempo de TV e o tempo sentado, ambos foram categorizados pela quantidade de horas diárias (valores de referência dos CS: $<2 \mathrm{~h} ; \geq 2 \mathrm{~h}$ e $<3 \mathrm{~h}$; $\geq 3 \mathrm{~h}$ e $<4 \mathrm{~h} ; \geq 4 \mathrm{~h}$ e $<5 \mathrm{~h} ; \mathrm{e} \geq 5 \mathrm{~h})$.

Em relação às variáveis contínuas, tem-se a idade (em anos completos), renda familiar mensal (valor absoluto recebido), valores referentes ao exame de sangue: HDL, Triglicérides e glicose sanguínea (em mg/dL), variáveis antropométricas (IMC, em $\mathrm{Kg} / \mathrm{m}^{2}$; e CC, em cm), e pressões sanguíneas (PAS e PAD, em mmHg).

Primeiramente, realizou-se uma análise descritiva dos dados, para obtenção das médias e desvio padrão das variáveis contínuas. Para isso, utilizou-se o Programa Estatístico IBM® SPSS® statistics versão 22.0.

Utilizou-se o programa Past (Paleontological Statistics Software Package for Education and Data Analysis) versão 2.17c para realização das análises MANOVA e ANOVA one-way (Análise de variância), para comparação dos diferentes pontos de corte dos comportamentos sedentários. Depois foi realizado o teste de Tukey, por conta da significância estatística, o qual foi representado na forma de BoxPlot; e, em seguida, a análise de componente principal (ACP) para analisar simultaneamente as diversas variáveis.

O presente estudo é parte da pesquisa intitulada "Perfil Epidemiológico dos Quilombolas baianos", autorizada pelo Comitê de Ética em Pesquisa com Seres Humanos da Universidade do Estado da Bahia, sob o parecer nº 1.386.019/2016. Os participantes foram voluntários, e assinaram ou forneceram a impressão digital no Termo de Consentimento Livre e Esclarecido.

\section{Resultados}

Nas 14 comunidades de remanescentes de quilombos visitadas, 850 moradores aceitaram participar do estudo; destes $520(61,2 \%)$ eram do sexo feminino. A média da idade foi de 44,7 $( \pm 16,95)$ anos e variou de 18 a 92 anos. Quanto aos grupos etários $684(80,5 \%)$ com idade de 18 a 59 anos e $166(19,5 \%)$ com 60 anos ou mais. A renda familiar mensal média foi $\mathrm{R} \$ 714,42( \pm 567,99)$ reais, variando de $\mathrm{R} \$ 30,00$ a $\mathrm{R} \$ 4.000,00$ e $579(79 \%)$ tinham renda familiar mensal menor ou igual a um salário mínimo (Tabela 1 e Tabela 2).

Nos quilombolas o tempo médio no CS tempo de TV foi $1,53 \mathrm{~h} /$ dia $( \pm 1,46)$ e variou de 0 a $13,33 \mathrm{~h} /$ dia. Enquanto que, 
para o tempo sentado a média foi $2,02 \mathrm{~h}( \pm 1,79)$, com variação de 0 a $12 \mathrm{~h} /$ dia (Tabela 2$)$.

Tabela 1. Distribuição das características socioeconômicas e demográficas da população quilombola da Microrregião de Guanambi, Bahia, Brasil, 2016.

\begin{tabular}{|c|c|c|}
\hline \multirow[t]{2}{*}{ Variável } & \multicolumn{2}{|c|}{ Amostra } \\
\hline & $\mathrm{n}$ & $\%$ \\
\hline \multicolumn{3}{|l|}{$\operatorname{Sexo}(n=850)$} \\
\hline Masculino & 330 & 38,8 \\
\hline Feminino & 520 & 61,2 \\
\hline \multicolumn{3}{|c|}{ Faixa Etária $(\mathrm{n}=850)$} \\
\hline 18-59 anos & 684 & 80,5 \\
\hline$\geq 60$ anos & 166 & 19,5 \\
\hline \multicolumn{3}{|c|}{ Renda Familiar Mensal (n=733) } \\
\hline$\leq 01 \mathrm{SM}^{*}$ & 579 & 79 \\
\hline$>01 \mathrm{SM}$ & 154 & 21 \\
\hline
\end{tabular}

*SM (salário mínimo) = R\$880,00. Fonte: Elaboração própria com dados da presente pesquisa.

Tabela 2. Descrição das medidas de dispersão e tendência central das variáveis: faixa etária, renda familiar mensal, tempo de TV e Tempo sentado da amostra da população quilombola da Microrregião de Guanambi, Bahia, Brasil, 2016.

Variável Média $\quad$ Valor (mínimo - máximo) desvio padrão

\begin{tabular}{lccc}
\hline Faixa Etária - em anos $(\mathrm{n}=850)$ & 44,47 & $18-92$ & $\pm 16,95$ \\
Renda Familiar Mensal - em Reais $(\mathrm{R} \$)(\mathrm{n}=733)$ & & $30,00-4.000,00$ & $\pm 567,99$ \\
& 714,42 & $0-93,33$ & $\pm 10,24$ \\
Tempo de TV - em horas/dia & 10,68 & $0-12$ & $\pm 1,76$ \\
Tempo Sentado - em horas/dia & 2,17 & & \\
\hline
\end{tabular}

Fonte: Elaboração própria com dados da presente pesquisa.

A Tabela 3 apresenta a distribuição dos CS por intervalos de tempo utilizados. Percebe-se que, no tempo gasto ao assistir TV, a maior prevalência $(69,7 \%)$ foi de pessoas que passam cinco ou mais horas de TV, enquanto no tempo sentado as que ficam menos de duas horas na posição sentada (54,5\%). Na distribuição de frequências entre os quilombolas que apresentaram elevados tempo de TV (Tabela 4), percebeu-se que, as maiores prevalências naqueles com três ou mais componentes da SM foi o intervalo de tempo $\geq 2 \mathrm{~h}$ e $<3 \mathrm{~h}$, em adultos $(47,4 \%)$ e nos idosos $(34,4 \%)$. 
Tabela 3. Distribuição dos comportamentos sedentários (tempo de TV e tempo sentado) por intervalo de tempo utilizado na população quilombola da Microrregião de Guanambi, Bahia, Brasil, 2016.

\begin{tabular}{lcc}
\hline & \multicolumn{2}{c}{ Variável n (\%) } \\
\cline { 2 - 3 } Intervalo de tempo* & Tempo de TV (n=746) & Tempo Sentado (n=791) \\
\hline$<2 \mathrm{~h}$ & $124(16,6)$ & $431(54,5)$ \\
$\geq 2 \mathrm{~h} \mathrm{e}<3 \mathrm{~h}$ & $28(3,8)$ & $161(20,4)$ \\
$\geq 3 \mathrm{~h} \mathrm{e}<4 \mathrm{~h}$ & $46(6,2)$ & $86(10,9)$ \\
$\geq 4 \mathrm{~h} \mathrm{e}<5 \mathrm{~h}$ & $28(3,8)$ & $49(6,2)$ \\
$\geq 5 \mathrm{~h}$ & $520(69,7)$ & $64(8,1)$ \\
\hline
\end{tabular}

*em horas/dia. Fonte: Elaboração própria com dados da presente pesquisa.

Nos adultos e idosos avaliados, nas análises MANOVA e ACP não houve resultados significativos ( $>0,05)$ entre o CS tempo sentado e os componentes da SM (HDL, CC, Triglicérides, IMC, Glicose sanguínea, PAS e PAD). Em relação ao comportamento tempo de TV, na MANOVA e na ACP não foram encontradas associações estatisticamente significativas com os componentes da SM (HDL, CC, Triglicérides, IMC, Glicose sanguínea, PAS e PAD) (p>0,05).

Contudo, em idosos, na ANOVA, observou-se que a CC apresentou resultado estatisticamente significante com diferença entre os tempos gastos assistindo TV de $4 \mathrm{~h}$ e $\geq 5 \mathrm{~h}$, pelo teste Turkey, por meio do qual, letras iguais não diferem estatisticamente entre si, e neste caso houve letras diferentes entre estes intervalos de tempo. Apesar de não ser explicada estatisticamente pela ACP, visualiza-se no BloxPlot que a CC deve ser considerada como importante variável associada a elevados tempos de TV na população avaliada (Figura 1).

Figura 1. BoxPlot da variação da Circunferência de cintura em relação aos diferentes tempos de TV em populações remanescentes de quilombos, Bahia, Brasil, 2016. Pelo teste de Tukey letras iguais não diferem estatisticamente entre sí com $\alpha=5 \%$.

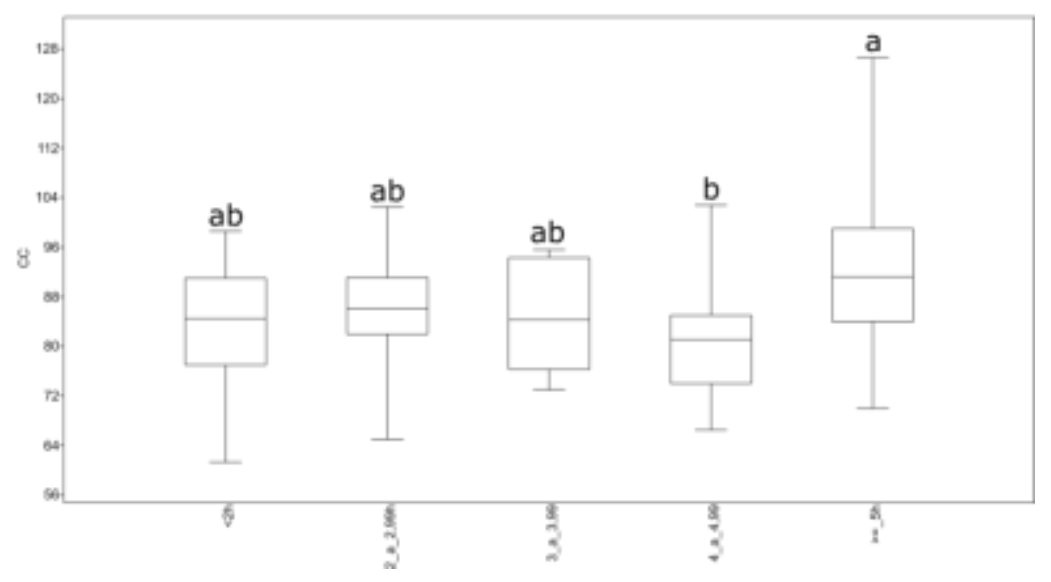

Fonte: Elaboração própria com dados da presente pesquisa.

\section{Discussão}

As características sociodemográficas e econômicas dos quilombolas são similares às encontradas em outras comunidades remanescentes de quilombos. Prevalecem quilombolas do sexo feminino (Araújo et al. 2017; Freitas, Rodrigues, 
Silva \& Nogueira, 2018), menor prevalência de idosos com mais de 60 anos (Araújo et al. 2017), e com renda familiar de até um salário mínimo (Freitas et al. 2018). Em estudo com quilombolas do estado do Pará, ao considerar adultos e idosos, foram encontrados dados divergentes visto que 35,9\% das famílias tinham renda mensal inferior a um salário mínimo (Araújo et al. 2017).

Os CS tempo de TV e tempo sentado têm sido utilizados para avaliar os CS. Entretanto, ainda não há um consenso acerca dos pontos de corte. Devido à falta de estudos que utilizam este instrumento para avaliar os CS fez-se necessário comparar os dados obtidos com os de estudos que utilizaram diferentes métodos para mensurar os CS.

A média diária do CS tempo de TV nos quilombolas da microrregião de Guanambi, Bahia foi baixa. Neste sentido, identificou-se estudo com homens afro-caribenhos, de meia idade e idosos, onde a média de tempo de TV foi $13.3 \pm 8.2$ horas/semana ( $\approx 2$ horas/dia) (Miljkovic et al. 2016). Médias maiores foram observadas em populações africanas e afroamericanas de um estudo longitudinal realizado em cinco países (Gana, África do Sul, Seychelles, Jamaica, e Estados Unidos) com idade de 25 a 45 anos, onde a média de tempo sedentário em counts foi $\geq 3 \mathrm{~h} /$ dia (Atiase et al. 2015). Em estudo com mulheres da África do Sul, a média do CS tempo sentado foi de 5h/dia (Micklesfield et al. 2017).

Estas disparidades nos tempos médios de CS podem ser, mesmo que parcialmente, explicadas pelas diversificadas rotinas comportamentais entre os diferentes grupos populacionais. Por exemplo, o estilo de vida de moradores de espaços rurais, como os quilombolas participantes desta análise, apresentarem indicadores importantemente diferentes daqueles de espaços urbanos (Rodrigues et al. 2020), resultantes de menor escolarização e mais recorrentes em populações rurais e negras, como as quilombolas.

$\mathrm{Na}$ amostra avaliada, 69,7\% quilombolas da microrregião de Guanambi, Bahia, permaneciam por um período $\geq$ 5horas/dia no CS tempo de TV. Ao avaliar adultos e idosos residentes em uma comunidade quilombola baiana foi identificado que a prevalência de tempo de $\mathrm{TV} \geq 2$ horas/dia foi de 36,2\% (Almeida, Mussi, Rodrigues \& Casotti, 2018). Deve-se considerar que a comparação entre estas comunidades quilombolas apresentam médias mais próximas. Isso pode ser explicado devido a características similares nos aspectos históricos, geográficos, culturais, econômicos e de acesso a aparelhos de TV. Estas características diferiram, assim, da população afro da região do Caribe, mesmo avaliando o tempo de TV (Miljkovic et al. 2016). Nota-se que, mesmo os CS terem sido avaliados de forma diferente, existe a presença elevada destes comportamentos em ambos os estudos.

Destarte, sabe-se que a dificuldade de acesso à renda pela população negra e rural brasileira, o que inclui as comunidades quilombolas, afeta a manutenção de estilo de vida mais saudável (Brasil, 2013), e pode interferir negativamente nos CS, ao considerar que estes componentes fazem parte desse estilo de vida desta população (Almeida, Casotti \& Sena, 2018; Almeida \& Casotti, 2020).

Na presente análise, no estrato dos idosos, dentre os componentes da SM, a CC se associou às diferenças em altos tempos de TV, o que indica este CS como capaz de contribuir no monitoramento e prevenção da SM. Destaca-se que, nas diretrizes da Organização Mundial de Saúde sobre atividade física e comportamento sedentário, foi identificado que o acúmulo de tecido adiposo armazenado na região central do corpo ocasionado pelos CS pode produzir células inflamatórias que são precursoras de doenças crônicas não transmissíveis (Meneguci et al. 2015). Neste contexto, o excesso do CS pode amplificar os, já reconhecidos, impactos mais intensos da obesidade em pessoas negras e mais velhas, especialmente quando em situação de vulnerabilidade, como já foi relatado em comunidades quilombolas (Santos, Melo, Santos \& Mussi, 2020).

Percebe-se que, tanto para o aumento dos CS quanto para o surgimento de componentes da SM, existem fatores que podem agravar estas condições em comunidades quilombolas, como por exemplo, estarem localizadas predominantemente em áreas rurais (Pereira et al. 2020) muitas vezes com difícil acesso geográfico em situação de má conservação e restritação no fornecimento de energia elétrica e outros serviços básicos (Almeida, Santos, Queiroz \& Mussi, 2020). 
Além disso, pode-se considerar que diversas comunidades sofrem com a ausência de infraestrutura de serviço de saúde, e com má cobertura do Programa de Agentes Comunitários de Saúde (Pereira et al. 2020) o que contribui para uma dificuldade no acesso aos serviços de saúde (Pereira et al. 2020; Almeida et al. 2018).

Nota-se que o racismo estrutural, persistente na sociedade brasileira, interfere nas políticas e serviços públicos de saúde (Almeida et al. 2018; Pereira \& Mussi, 2020), que por sua vez acabam modificando negativamente o estilo de vida dos quilombolas, e consequentemente contribui para o aumento de CS e condições de saúde debilitadas (Almeida, Casotti \& Sena, 2017), inclusive um estudo considerou o sofrimento psíquico como relacionado, dentre outras coisas, com as iniquidades sociais (Dimenstein et al. 2020).

Relata-se que permanecer longo período de tempo em CS reduz o consumo de glicose pelos músculos esqueléticos, aumenta a resistência à insulina e atrofia muscular. Com isso, haveria redução da utilização de energia pelos músculos ativos e o fígado aumentaria a produção de lipídios, devido à inativação da enzima lipoproteína lipase e, consequentemente, o armazenamento de tecido adiposo na região central do corpo (Meneguci et al. 2015).

O acúmulo de tecido adiposo pode desencadear problemas diversos à saúde, tais como obesidade, risco metabólico, níveis elevados de glicose e hipertrigliceridemia (Micklesfield et al. 2017; Atiase et al. 2015; Dickie et al. 2016). Vale destacar que permanecer por período de tempo excessivo em CS pode ser associado a patologias relacionadas ao aparelho cardiovascular, como hipertensão arterial (Cordovil \& Almeida, 2018), risco de acidente vascular cerebral isquêmico (Owolabi et al. 2017) e risco de mortalidade por todas as causas (Matthews et al. 2014).

Neste sentido, em estudo com idosos de Florianópolis, Brasil, ao avaliar o CS por meio de acelerômetro, verificou-se que aqueles com CS tiveram maior CC média quando comparados aos seus pares (Figueiró et al. 2019). Outro estudo que avalia os CS, também com populações não quilombolas, mostrou associação deste comportamento com a CC (Rezende et al. 2014).

Em mulheres quilombolas de Palmas, Paraná, Brasil, com média de idade de 38 anos (DP $\pm 16,554$ ), a CC foi um dos principais parâmetros antropométricos, tanto para o excesso de peso corporal, quanto para a condição de risco aumentado de desenvolver alguma doença cardiovascular (Vieira et al. 2019). Alguns estudos identificaram riscos à saúde de quilombolas, e destacaram a CC como um dos principais preditores de excesso de peso (Cordovil \& Almeida, 2018; Vieira et al. 2019) e de risco cardiovascular nesta população (Cordovil \& Almeida, 2018; Vieira et al. 2019; Alcântara et al. 2019).

Em uma revisão sistemática sobre CS e saúde de pessoas com mais de 60 anos de idade, observou-se que em alguns estudos a CC foi associada aos CS. Dentre as associações, encontraram-se relações entre: tempo sentado e aumento do risco de obesidade abdominal; tempo de TV e tempo de lazer associado positivamente a CC; tempo de TV excessivo e relação cinturaquadril elevada; maior tempo gasto em CS e CC elevada (Rezende et al. 2014).

No estudo ELSA-BRASIL que investigou fatores associados aos CS em 13.765 pessoas de ambos os sexos, por meio de regressão logística, encontrou associação positiva com a obesidade abdominal e o tempo sentado $\geq 8 \mathrm{~h} /$ dia (Pitanga et al. 2018). Assim, deve-se ter cautela ao comparar os resultados destes estudos, tendo em vista que utilizaram diferentes populações, formas de mensurar e categorizar os CS. Entretanto, estes achados devem ser vistos como um alerta, uma vez que os mecanismos fisiológicos dos CS são similares e podem causar problemas de saúde parecidos.

Além destas associações, estudo realizado na Amazônia Legal, no município de Barra do Garça, com adultos de ambos os sexos, identificou que as mulheres se envolviam mais do que os homens em atividades sedentárias, tais como, assistir TV e usar computador. O estudo ainda mostrou que elas tiveram maior prevalência de CC (70,73\%) do que os homens $(35,35 \%)$. Os autores chamam a atenção para os resultados encontrados, visto que elevados valores de CC estão associados a um maior risco cardiovascular e metabólico (Spanhol \& Bucalen-Ferrari, 2016).

Como limitação da presente pesquisa, pode-se considerar que as entrevistas realizadas estão susceptíveis a viés de 
memória. Entretanto, a representatividade da amostra pode ser considerada um fator positivo para dirimir possíveis erros. Além disso, sabe-se que ainda não existe um consenso na definição, mensuração e avaliação dos CS (Gibbs et al. 2015), o que pode justificar a utilização de diferentes intervalos de tempo para CS na presente pesquisa.

Vale ressaltar que existe uma importância social em se investigar de forma científica a associação de CS com componentes da SM nesta população, que é vulnerável em diversos aspectos, principalmente social (Pauli et al. 2019), socioeconômico e ambiental (Araújo et al. 2019). Pois, destaca-se a dificuldade em identificar publicações com outros parâmetros que podem ser associados tanto aos CS quanto a SM em quilombolas. Tal fato fez com que fosse, por vezes, utilizados estudos com populações diferentes das quilombolas.

\section{Considerações Finais}

Na população quilombola da região participante da investigação não foi identificada associação entre os diferentes tipos de CS com os componentes da SM. Todavia, no estrato dos idosos, a CC foi o componente da SM que mostrou diferenças significativas entre os tempos 4 h e os com cinco ou mais horas ao assistir TV.

Neste sentido, sugere-se que a CC pode ser influenciada pelo tempo de TV elevado, o que contribui para a promoção de condições desfavoráveis à saúde da população remanescente de quilombos da Microrregião pesquisada. A relação deste CS com a CC precisa ser aprofundada, tendo em vista que esta variável quando em valor elevado, pode ser relacionada com a SM, e com outras condições de saúde como, por exemplo, aumento do risco cardiovascular e obesidade.

Ao desenvolver investigações com esse perfil populacional é importante considerar a participação das condições de vulnerabilidade, calcada no racismo institucional, que amplia as desigualdades nas condições de vida e de saúde. Pois, essas condições, representam permanências sociohistóricas impostas a esta população, que resultam em situações desfavoráveis com reflexos negativos multidimensionais.

Assim, em pesquisas futuras, sugere-se que seja considerada a relação entre as viariáveis de CS e os altos níveis de atividade física. E, ainda que as análises sejam estratificadas por sexo. Estas sugestões poderão ampliar a compreensão de possíveis associações entre os CS, tempo sentado e tempo de TV, com os componentes da SM.

\section{Referências}

Alcântara, T. G., Souza, T., Santana, A., Carmo, T., Gomes, T., \& Menês, L., Estrela, A. R. R., Ferraz, H. R., Sá, M. S. L. C., Cairo, C. H. S., Chaves, A. C. T. A., Souza, C. L., Teles, M. F. \& Queiroz, R. F. (2019). Prevalence of cardiovascular risk factors in two Brazilian quilombola communities in Southwest Bahia State. International Journal Of Advanced Engineering Research And Science, 6(12), 297-302. doi: 10.22161/ijaers.612.28 (2019). Prevalence of cardiovascular risk factors in two Brazilian quilombola communities in Southwest Bahia State. International Journal of Advanced Engineering Research and Science, 6(12), 297-302.

Almeida, C. B., Mussi, R. F. F., Rodrigues, D. N. \& Casotti, C. A. (2018). Determinantes sociodemográficas do comportamento sedentário em adultos quilombolas. Anais do Convención Internacional de Salud, Cuba Salud $2018 . \quad$ Havana, Cuba. http://www.convencionsalud2017.sld.cu/index.php/connvencionsalud/2018/paper/view/941/1216

Almeida, C. B., Santos, A. S., Vilela, A. B. A. \& Casotti, C. A. (2019). Reflexão sobre o controle do acesso de quilombolas à saúde pública brasileira. Avances em Enfermeria, 37(1), 92-103.

Almeida, C. B. \& Casotti, C. A. (2020). Estilo de vida da população afrodescendente: revisão integrativa. Journal of Nursing and Health, 10, e20104012.

Almeida, C. B. \& Casotti, C. A. (2021). Revisão integrativa: comportamentos sedentários, síndrome metabólica e seus componentes em populações quilombolas. International Journal of Development Research, 11(1), 43657-43661.

Almeida, C. B., Casotti, C. A. \& Sena, E. L. S. (2018). Reflexões sobre a complexidade de um estilo de vida saudável. Avances em Enfermeria, 36(2), 220229.

Almeida Filho, N. \& Barreto, M. L. (2017). Epidemiologia \& saúde: fundamentos, métodos, aplicações. Rio de Janeiro: Guanabara Koogan.

Almeida, I. L. S.; Santos, S. R.; Queiroz, B. M. \& Mussi, R. F. F. (2020). Estilo de vida, morbidades e multimorbidade em Quilombolas adultos. ABCS Health Sciences, 45, 1325. 
Aragão, J. A., Bós, A. J. G. \& Souza, G. C. (2014). Síndrome metabólica em adultos e idosos de comunidades quilombolas do centro-sul do Piauí, Brasil. Estudos Interdisciplinares sobre o Envelhecimento, 19(2), 501-512.

Araújo, A., Anjos, D., Silva, R., Santos, M., Martins, C. \& Almeida, R. (2017). Análise socioeconômica de agricultores da comunidade quilombola do Abacatal, Ananindeua, Estado do Pará, Brasil. Biota Amazônia, 7(1), 30-37.

Araújo, R. L. M. S., Araújo, E. M., Silva, H. P., Santos, C. A. S. T., Nery, F. S., Santos, D. B. \& Souza, B. L. M. (2019). Condições de vida, saúde e morbidade de comunidades quilombolas do semiárido baiano, Brasil. Revista Baiana de Saúde Pública, 43(1), 226-246.

Atiase, Y., Farni, K., Plange-Rhule, J., Luke, A., Bovet, p., Forrester, T. G., Lambert, V., Levitt, N. S., Kliethermes, S., Cao, G., Durazo-Arvizu, R. A., Cooper, R. S. \& Dugas, L. R. (2015). A comparison of indices of glucose metabolism in five black populations: data from modeling the epidemiologic transition study (METS). BMC Public Health, 15, 895

Brasil. (2016). Fundação Palmares. Brasília: Fundação Palmares. http://www.palmares.gov.br

Brasil. (2013). Secretaria de Gestão Estratégica e Participativa. Departamento de Apoio à Gestão Participativa. Política Nacional de Saúde Integral das Populações do Campo e da Floresta. Brasília: Ministério da Saúde.

Bull, F. C., Al-Ansari, S. S., Biddle, S., Borodulin, K., Buman, M. P., Cardon, G., Carty, C., Chaput, J. P., Chastin, S., Chou, R., Dempsey, P. C., DiPietro, L., Ekelund, U., Firth, J., Friedenreich, C. M., Garcia, L., Gichu, M., Jago, R., Katzmarzyk, P. T., Lambert, E., ... Willumsen, J. F. (2020). World Health Organization 2020 guidelines on physical activity and sedentary behaviour. British journal of sports medicine, 54(24), 1451-1462.

Cordovil, Y. F. \& Almeida, S. S. (2018). Variaveis antropometricas e fatores de risco cardiovascular associados em quilombolas marajoaras. Revista Brasileira de Obesidade, Nutrição e Emagrecimento, 12(71), 406-415.

Dickie, K., Micklesfield, L. K., Chantler, S., Lambert, E. V. \& Goedecke, J. H. (2016). Cardiorespiratory Fitness and Light-Intensity Physical Activity Are Independently Associated with Reduced Cardiovascular Disease Risk in Urban Black South African Women: A Cross-Sectional Study. Metabolic syndrome and related disorders, 14(1), 23-32.

Dimenstein, M., Belarmino, V., Martins, M., Dantas, C., Macedo, J., Leite, J. \& Alves Filho, A. (2020). Desigualdades, racismos e saúde mental em uma comunidade quilombola rural. Amazônica - Revista de Antropologia, 12(1), 205-229.

Figueiró, T. H., Arins, G., Santos, C., Cembranel, F., Medeiros, P. A., d'Orsi, E. \& Rech, C. R. (2019). Association of objectively measured sedentary behavior and physical activity with cardiometabolic risk markers in older adults. PloS one, 14(1), e0210861.

Freitas, I. A., Rodrigues, I. L. A., Silva, I. F. S. \& Nogueira, L. M. V. (2018). Perfil sociodemográfico e epidemiológico de uma comunidade quilombola na Amazônia Brasileira. Revista Cuidarte, 9(2), 2187-2200.

Gibbs, B. B., Hergenroeder, A. L., Katzmarzyk, P. T., Lee, I. M. \& Jakicic, J. M. (2015). Definition, measurement, and health risks associated with sedentary behavior. Medicine and science in sports and exercise, 47(6), 1295-1300.

Matos, F., Vasconcelos, L.R., Rocha, S.V. \& Mussi, R.F. (2021). Subdiagnóstico da Hipertensão Arterial em adultos quilombolas de região baiana, Brasil. Research, Society and Development, 10.

Matthews, C. E., Cohen, S. S., Fowke, J. H., Han, X., Xiao, Q., Buchowski, M. S., Hargreaves, M. K., Signorello, L. B. \& Blot, W. J. (2014). Physical activity, sedentary behavior, and cause-specific mortality in black and white adults in the Southern Community Cohort Study. American journal of epidemiology, 180(4), 394-405.

Melo, A. C. M. \& Garcia, L. P. (2019). Fatores associados a agressões por desconhecidos entre jovens do sexo masculino atendidos em serviços de urgência e emergência: estudo de casos e controles. Ciência \& Saúde Coletiva, 24(8), 2825-2834.

Meneguci, J., Santos, D. A. T., Silva, R. B., Santos, R. G., Sasaki, J. E., Tribess, S., Damião, R. \& Júnior, J. S. V. (2015). Comportamento sedentário: Conceito, implicações fisiológicas e os procedimentos de avaliação. Motricidade, 11(1), 160-174.

Micklesfield, L. K., Munthali, R. J., Prioreschi, A., Said-Mohamed, R., van Heerden, A., Tollman, S., Kahn, K., Dunger, D. \& Norris, S. A. (2017). Understanding the Relationship between Socio-Economic Status, Physical Activity and Sedentary Behaviour, and Adiposity in Young Adult South African Women Using Structural Equation Modelling. International journal of environmental research and public health, 14(10), 1271.

Miljkovic, I., Kuipers, A. L., Cvejkus, R., Bunker, C. H., Patrick, A. L., Gordon, C. L. \& Zmuda, J. M. (2016). Myosteatosis increases with aging and is associated with incident diabetes in African ancestry men. Obesity (Silver Spring, Md.), 24(2), 476-482.

Mussi, R. F.F., Queiroz, B.M. \& Petróski, E. L.. (2018). Excesso de peso e fatores associados em quilomboras do médio São Francisco baiano, Brasil. Ciência \& Saúde Coletiva, 23(4), 1193-1200.

Mussi, R. F.F. \& Petróski, E. L. (2019). Síndrome metabólica e fatores associados em quilombolas baianos, Brasil. Ciência \& Saúde Coletiva, 24(7), 24812490 .

Mussi, R. F. F., Teixeira Mussi, L. M. P., Rodrigues, D. N., Almeida, C. B., Teixeira, E.P. \& Figueiredo, A. C. M. G. (2020a). Inquérito de Saúde em População Quilombola Baiana: relato de uma experiência em pesquisa epidemiológica. Saúde e Pesquisa, 13(3), 675-685.

Mussi, R. F. F., Figueireido, A. C. M. G., Queiroz, B. M., Petroski, E. L., Rodrigues, D. N. \& Almeida, C. B. (2020b). Simultaneidades dos componentes da síndrome metabólica em adultos quilombolas baianos, Brasil. Nursing (São Paulo), 23(261), 3583-3588.

Owolabi, M., Sarfo, F., Howard, V. J., Irvin, M. R., Gebregziabher, M., Akinyemi, R., Bennett, A., Armstrong, K., Tiwari, H. K., Akpalu, A., Wahab, K. W., Owolabi, L., Fawale, B., Komolafe, M., Obiako, R., Adebayo, P., Manly, J. M., Ogbole, G., Melikam, E., Laryea, R., ... SIREN-REGARDS Collaboration 
Research, Society and Development, v. 11, n. 1, e30211125001, 2022

(CC BY 4.0) | ISSN 2525-3409 | DOI: http://dx.doi.org/10.33448/rsd-v11i1.25001

(Stroke Investigative Research and Educational Network-Reasons for Geographic and Racial Differences in Stroke) (2017). Stroke in Indigenous Africans, African Americans, and European Americans: Interplay of Racial and Geographic Factors. Stroke, 48(5), 1169-1175.

Pauli, S., Bairros, F. S., Nunes, L. N. \& Neutzling, M. B. (2019). Prevalência autorreferida de hipertensão e fatores associados em comunidades quilombolas do Rio Grande do Sul, Brasil. Ciência \& Saúde Coletiva, 24(9), 3293-3303.

Pereira, R., Mussi, R. \& Rocha, R. (2020). Acesso e Utilização dos Serviços de Saúde por quilombolas contemporâneos baianos. Revista da Associação Brasileira de Pesquisadores/as Negros/as (ABPN), 12(31).

Pereira, R. N. \& Mussi, R. F. F. (2020). Acesso e utilização dos serviços de saúde da população negra quilombola: uma análise bibliográfica. ODEERE, 5(10), 280-303.

Pitanga, F. J. G., Matos, S. M. A., Almeida, M. da C., Griep, R. H., Viana, M. C., Melo, E. C. P. \& Aquino, E. (2018). Factors associated with sedentary behavior among ELSA-Brasil participants: ecological model. Revista Brasileira de Atividade Física \& Saúde, 23, 1-8.

Rezende, L. F. M., Rey-López, J. P., Matsudo, V.K. \& Carmo L. O. (2014). Sedentary behavior and health outcomes among older adults: a systematic review. BMC Public Health, 14, 333.

Rodrigues, D. N., Mussi, R. F. F., Almeida, C. B., Nascimento Junior, J. R. A., Moreira, S. R. \& Carvalho, F. O. (2020). Determinantes sociodemográficos associados ao nível de atividade física de quilombolas baianos, inquérito de 2016. Epidemiologia e Serviços de Saúde, 29(3), e2018511.

Santos, A. G., Melo, F. T., Santos, M. S. \& MUSSI, R. F. F. (2020). Fatores Associados à Obesidade em Adultos Quilombolas baianos. Revista Brasileira de Obesidade, Nutrição e Emagrecimento, 14(85), 230-240.

Secretaria de Políticas de Promoção da Igualdade Racial da Presidência da República (SEPPIR). (2010). Comunidades Quilombolas Brasileiras Regularização Fundiária e Políticas Públicas. Brasília.

SOARES, D.A. \& BARRETO, S.M. (2015). Indicadores nutricionais combinados e fatores associados em população Quilombola no Sudoeste da Bahia, Brasil. Ciência \& Saúde Coletiva, 20(3), 821-832.

Sociedade Brasileira de Patologia Clínica/Medicina Laboratorial. (2014). Recomendações da Sociedade Brasileira de Patologia Clínica/Medicina Laboratorial (SBPC/ML): coleta e preparo da amostra biológica. Barueri: Manole.

Sousa, B. C., Medeiros, D. S., Curvelo, M. H. S., Silva, E. K. P., Teixeira, C. S. S., Bezerra, V. M., Souzas, R. \& Leite, A. J. M. (2019). Hábitos alimentares de adolescentes quilombolas e não quilombolas da zona rural do semiárido baiano, Brasil. Ciência \& Saúde Coletiva, 24(2), 419-430.

Spanhol, R. C. \& Bucalen-Ferrari, C. K. (2016). Obesity and lifestyle risk factors among an adult population in Legal Amazon, Mato Grosso, Brazil. Revista de Salud Publica, 18(1), 26-36.

Stewart, A. D., Marfell-Jones M., Olds T. \& Ridder H. (2011). International Standards for Anthropometric Assessment. New Zealand: ISAK.

Teixeira Mussi, L. M. P., Mussi, R. F. F. \& Araujo, A. G. (2020). Negligência/Negação a Escolarização em Quilombos da Região de Guanambi/Bahia. Veredas, 3(5), 80-103.

Vieira, V. K., Lazarotto, A. K., Antes, D. C., Silva, J. C., Motter, N.S., Teló, V. L. B., Treco, I. C. \& Lucio, L. C. (2019). Prevalência e preditores do excesso de peso e do risco cardiovascular em mulheres quilombola de Palmas, PR. Brazilian Journal of Development, 5(12), 32277-32299. 